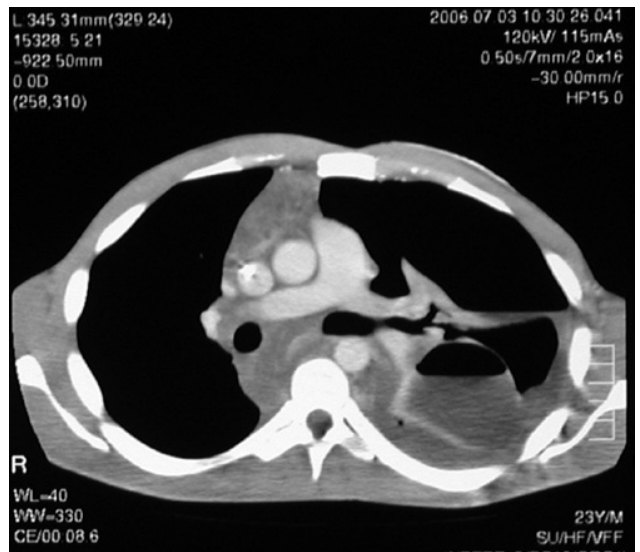

Figure 2. Mediastinal involvement and loculated cystic spaces of the left pleural cavity.

of the patient. Therefore, treatment cannot be standardized and decision-making is customized to the particularities of the case. ${ }^{4}$

Generalized lymphangiomatosis management can be challenging. Surgical treatment is indicated for complications (such as chylopericardium and chylothorax) or for biopsy. An important issue for management of chylous effusions is that there is not one specific source of chyle that can be stopped through the thoracic duct ligation; instead, there is a wide abnormal network of lymphatic channels. However, the best results have been reported with pleurectomy, with or without thoracic duct ligation, whereas thoracic duct ligation alone was unsuccessful in all cases. Fibrin glue application may help control lymphatic leakage from exposed surfaces after pleurectomy. ${ }^{2}$ Other treatment options include interferon alfa and systemic chemotherapy with limited success, and radiation in fractioned dose has been promising for patients with extensive mediastinal disease. ${ }^{5}$

\section{References}

1. Faul JL, Berry GJ, Colby TV, Ruoss SJ, Walter MB, Rosen GD, et al. Thoracic lymphangiomas, lymphangiectasis, lymphangiomatosis, and lymphatic dysplasia syndrome. Am J Respir Crit Care Med. 2000;161: 1037-46.

2. Canil K, Fitzgerald P, Lau G. Massive chylothorax associated with lymphangiomatosis of the bone. J Pediatr Surg. 1994;29:1186-8.

3. Takahashi K, Takahashi H, Maeda K, Homma S, Uekusa T, Dambara T, et al. An adult case of lymphangiomatosis of the mediastinum, pulmonary interstitium and retroperitoneum complicated by chronic disseminated intravascular coagulation. Eur Respir J. 1995;8:1799-802.

4. Zisis C, Rontogianni D, Charalambous E, Bellenis I. Lymphangiomatous hamartoma: cause or bystander of the isolated chylopericardium? J Thorac Cardiovasc Surg. 2005;130:1201-2.

5. Rostom AY. Treatment of thoracic lymphangiomatosis. Arch Dis Child. 2000;83:138-9.

\title{
Giant cavernous hemangioma of the distal esophagus treated with esophagectomy
}

\author{
Anthony W. Kim, MD, Robert J. Korst, MD, Jeffrey L. Port, MD, Nasser K. Altorki, MD, and Paul C. Lee, MD, New York, NY
}

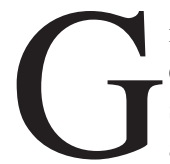
iant cavernous hemangiomas are rare tumors of the esophagus. In 1976, Feist, Talley, and $\mathrm{Hunt}^{1}$ described an upper esophageal hemangioma as "giant"; it was preoperatively $4 \times 7 \mathrm{~cm}$ and pathologically $12.0 \times 5.0 \times 2.5 \mathrm{~cm}$ in size. We present the case of an asymptomatic 68-year-old woman who had a giant hemangioma of the distal esophagus. She underwent an esophagectomy with

From the Department Cardiothoracic Surgery, Weill Medical College of Cornell University, New York, NY.

Received for publication Jan 16, 2007; accepted for publication Feb 12, 2007.

Address for reprints: Anthony W. Kim, MD, Rush University Medical Center, 1725 W Harrison St, Suite 774, Chicago, IL 60612 (E-mail: Anthony_kim@rush.edu).

J Thorac Cardiovasc Surg 2007;133:1665-7

0022-5223/\$32.00

Copyright $\odot 2007$ by The American Association for Thoracic Surgery doi:10.1016/j.jtcvs.2007.02.004 a gastric pull-up via a left thoracotomy. Her recovery was unremarkable and she is doing well at 4 months' follow-up.

\section{Clinical Summary}

A 68-year-old asymptomatic woman was found to have an abnormality on a chest radiograph before cataract surgery. A follow-up computed tomographic (CT) scan demonstrated a large lobulated distal esophageal mass with multiple small calcifications (Figure 1). Endoscopy was negative for mucosal lesion. A positron emission tomographic (PET) scan revealed that the mass had a standardized uptake value of 2.0. A transthoracic biopsy of the lesion demonstrated only blood and fibrinated material. Magnetic resonance imaging (MRI) reidentified the lobulated soft tissue mass in the distal esophagus and described it as a fusiform lesion involving the esophageal wall, causing narrowing of the lumen. Neither mediastinal invasion nor lymphadenopathy was noted. On the basis of the soft tissue signal and the smooth appearance, the lesion was thought to be a large leiomyoma.

The patient was brought to the operating room where she underwent a left posterolateral thoracotomy through the sixth 


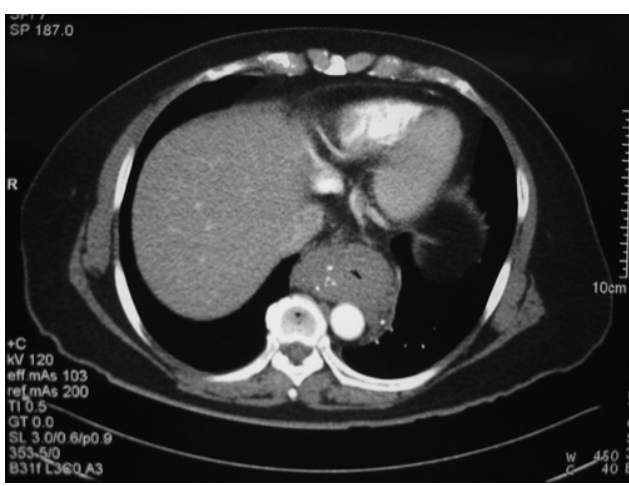

Figure 1. Computed tomographic scan demonstrating a large circumferential distal esophageal hemangioma with multiple small calcifications.

intercostal space. The mass was soft, boggy, and very vascular, and it extended from the inferior pulmonary vein to the gastroesophageal junction (Figure 2). There was concern of malignancy owing to the macroscopic appearance of pericardial invasion. The esophagus was mobilized from below the aortic arch to the gastroesophageal junction. Included in this dissection was an en bloc portion of the pericardium that appeared to be invaded. The diaphragm was subsequently opened circumferentially and the stomach was fully mobilized. The stomach was delivered through the hiatus and divided along the lesser curvature. The esophagus was then divided below the aortic arch. The stomach was then tubularized and a handewn anastomosis was then performed with 3-0 polydioxanone sutures (PDS; Ethicon, Inc, Somerville, NJ). The diaphragmatic defect was then reapproximated and the left thoracotomy was closed after placement of a $28 \mathrm{~F}$ chest tube. Final pathologic examination revealed an $8.5 \times 6.5 \times 4.0$-cm cavernous hemangioma with calcified thrombi, involving the esophagus and proximal stomach.

Postoperatively, the patient's course was unremarkable. On postoperative day 5 , she had a barium esophagogram that was negative for an anastomotic leak. She was subsequently advanced to a soft mechanical diet. She was discharged to her home on postoperative day 8. At 4 months' follow-up the patient was doing well.

\section{Discussion}

Generally, patients with cavernous hemangiomas of the esophagus have presented with hematemesis that ranges from self-limiting to life-threatening or with varying degrees of dysphagia. ${ }^{1-5}$ An asymptomatic presentation, however, is not uncommon. ${ }^{3,4}$ Endoscopically, hemangiomas usually are described as submucosal lesions that appear anywhere along the esophagus, are bluish, compressible, and protruding. ${ }^{3,4}$ They can also have atypical appearances including a reddish discoloration, a normal-appearing mucosa, or an ulcerated appearance. ${ }^{1}$ Barium esophagogram may initially identify a filling or luminal defect, but CT scans may better delineate the lesion itself. The intact mucosa often associated with this submucosal lesion may be missed on endoscopy even in the presence of a large intraesophageal tumor. ${ }^{1}$ On CT scans, these tumors appear homogeneous with small calcifications

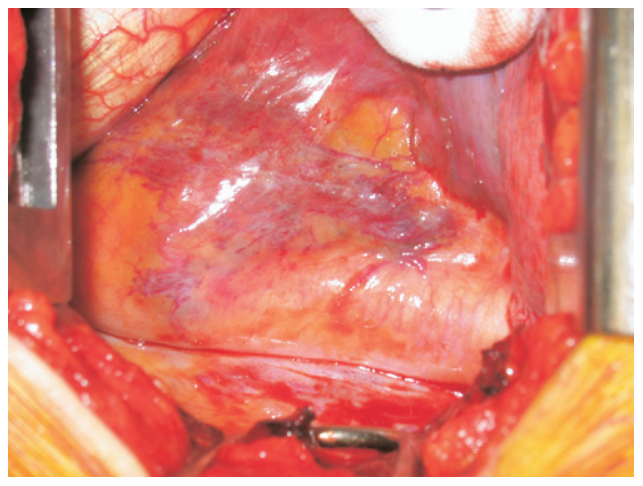

Figure 2. Left thoracotomy. Initial exploration revealed a distal esophageal cavernous hemangioma with extensive vascular structures emanating circumferentially from the lesion, anterior to the aorta, and involving the pericardium.

or phleboliths. ${ }^{4}$ Although endoscopic ultrasound was not performed in this patient, early reports have detailed the usefulness of this procedure in further characterizing this lesion. Sonographically, hemangiomas range from anechoic to hypoechoic to isoechoic and involve the mucosal and submucosal layers with an intact muscularis propria. ${ }^{4,5}$ Endoscopic ultrasound can also confirm the absence of continuity with major blood vessels. ${ }^{4-5}$

In the patient presented in this report, magnetic resonance imaging and angiography (MRI/MRA) were used preoperatively to help distinguish this lesion. Characteristic MRI findings have included an isointense signal from the mass on T1-weighted images and a high signal on T2-weighted images. ${ }^{3,4}$ Other reports have described atypical features such as a high-intensity signal from the mass on both T1- and T2-weighted images. ${ }^{4}$ In this specific patient, the T1-weighted images demonstrated a predominantly soft tissue type signal. The T2-weighted images demonstrated the soft tissue signal type with areas of small hyperintense signals and small areas of hypointense signals. MRA demonstrated the absence of any dominant vessels extending into or toward the mass.

Despite recent advances, arriving at the definitive diagnosis of a hemangioma preoperatively can be elusive when using noninvasive modalities. Endoscopic biopsy of the intraesophageal tumor is commonly used to obtain a preoperative histologic diagnosis. Often a concern of hemorrhage is the reason cited for not performing an endoscopic biopsy, but in reality this is a rare occurrence. ${ }^{5}$ In lieu of the more frequently described endoscopic biopsy, a transthoracic biopsy was performed in the patient in this current report. Unfortunately, the tissue obtained in this case yielded an inconclusive result. A review of the current literature does not describe the routine use of transthoracic needle biopsy in the diagnosis of this type of tumor.

When preoperative studies are inconclusive, surgical resection is often indicated both for diagnosis and to rule out malignant disease. ${ }^{5}$ In this case report, the tumor was noted to be large on preoperative imaging studies and locally invasive on initial exploration. These features suggested a malignant process and therefore a resection was indicated. Additionally, the potential risk of bleeding from the vascular tumor, if left unresected, warranted resection. ${ }^{3,5}$ 
The modern literature suggests that endoscopic resection is adequate in the treatment of this tumor. Submucosal esophageal cavernous hemangiomas have been successfully resected by the endoscopic polypectomy or mucosal resection techniques. ${ }^{4,5}$ Alternatively, other ablative techniques, such as laser therapy, also have been described in the treatment of cavernous hemangiomas. ${ }^{3}$ These modalities, however, are used in the treatment of lesions that are smaller than the one described in the present patient.

Before modern endoscopic instrumentation, limited local resection in the form of a polypectomy or enucleation was the accepted or even favored method of treatment. Approaches to a limited resection have classically been through a thoracotomy. ${ }^{2}$ Recently, thoracoscopic removal has also been described in the treatment of these lesions with the simultaneous use of a flexible endoscope. $^{2}$ This obviously is possible only if the lesion is small enough for this type of resection. Esophagectomy has rarely been described in the treatment of this lesion, but this is because, in general, the majority of cavernous hemangiomas of the esophagus reported are on the order of 2 to $3 \mathrm{~cm} .^{3}$ The hemangioma described in this report was giant, and concerns of malignancy and potential hemorrhage warranted an esophagectomy.

\section{References}

1. Feist K, Talley NA, Hunt DR. Hemangioma of the esophagus: an unusual cause of upper gastrointestinal bleeding. JAMA. 1976;235:1146-7.

2. Ramo OJ, Salo JA, Bardini R, Nemlander AT, Farkkila M, Mattila SP. Treatment of submucosal hemangioma of the esophagus using a simultaneous video-assisted thoracoscopy and esophagoscopy: description of a new minimally invasive technique. Endoscopy. 1997;29:S27-8.

3. Shigemitsu K, Naomoto Y, Yamatsuji T, Ono K, Aoiki H, Haisa M, et al. Esophageal hemangioma successfully treated by fulguration using potassium titanyl phosphate/yttrium aluminum garnet (KTP/YAG) laser: a case report. Dis Esoph. 2000;13:161-4.

4. Tominaga K, Arakawa T, Ando K, Umeda S, Shiba M, Suzuki N, et al. Oesophageal cavernous haemangioma diagnosed histologically, not by endoscopic procedures. J Gastroenterol Hepatol. 2000;15:215-9.

5. Cantero D, Yoshida T, Ito M, Suzumi M, Tada M, Okita K. Esophageal hemangioma: endoscopic diagnosis and treatment. Endoscopy. 1994;26: 250-3.

\title{
Primary Ewing sarcoma invading the heart: Resection and reconstruction
}

\author{
Subroto Paul, MD, ${ }^{\text {a }}$ Tharumenthiran Ramanathan, MD, PhD, FRACS, ${ }^{\text {b }}$ Daniel M. Cohen, MD, a Abraham Lebenthal, MD, ${ }^{a}$ \\ Lambros Zellos, MD, ${ }^{a}$ Sary F. Aranki, MD, and David J. Sugarbaker, MD, ${ }^{a}$ Boston, Mass
}

\section{Clinical Summary}

$\mathrm{W}$ e describe the case history of a 14-year-old boy who came to his pediatrician with a cough. Chest radiograph revealed a large mediastinal mass. Workup with chest computed tomography (CT) and magnetic resonance imaging (MRI) revealed that the mass had invaded the right pulmonary parenchyma, both atria, and the superior vena cava (SVC) (Figure 1, A). CT-guided biopsy revealed the tumor to be Ewing sarcoma/primitive neuroectodermal tumor. The patient underwent sarcoma-based chemotherapy with 18 weeks of vincristine, ifosafamide, doxorubicin (Adriamycin), and etoposide followed by 2 additional weeks of vincristine, doxorubicin, and isofamide. Repeat imaging revealed the mass to be downsized by chemotherapy

From the Divisions of Thoracic Surgery ${ }^{\mathrm{a}}$ and Cardiac Surgery, ${ }^{\mathrm{b}}$ Department of Surgery, Brigham and Women's Hospital, Boston, Mass.

Received for publication Jan 6, 2007; accepted for publication Feb 7, 2007.

Address for reprints: David J. Sugarbaker, MD, Brigham and Women's Hospital, Division of Thoracic Surgery, 75 Francis St, Boston, MA 02115 (E-mail: dsugarbaker@partners.org).

J Thorac Cardiovasc Surg 2007;133:1667-9

$0022-5223 / \$ 32.00$

Copyright () 2007 by The American Association for Thoracic Surgery doi:10.1016/j.jtcvs.2007.02.003
(Figure 1,B). Bone scan, abdominal CT, and positron emission tomography-CT did not reveal any metastases. The patient was referred for surgery. Preoperative workup included pulmonary function tests (forced expiratory volume in 1 second $=2.59$ [67\%], forced vital capacity $=3.00[67 \%]$ ), a quantitative ventilation/perfusion scan (quantitative perfusion [left lung] = $81 \%$; quantitative perfusion [right lung] $=19 \%$ ), and an echocardiogram (ejection fraction $=65 \%$ with normal left and right ventricular function).

A median sternotomy was performed and both pleurae and the pericardium were entered. The mass involved the distal third of the SVC, the free wall of the right and left atria, the interatrial septum, and the right lung, but was deemed resectable. The patient was placed on cardiopulmonary bypass via aortic and bicaval cannulation and cooled to $28^{\circ} \mathrm{C}$ with the heart arrested with antegrade blood cardioplegia. An en bloc resection of the lower third of the SVC, the free wall of the right and left atria along with the interatrial septum, and a right pneumonectomy were performed (Figure 2, A). Frozen section margins were negative for tumor involvement. The interatrial septum and free wall of the right atrium were reconstructed with glutaraldehyde-treated pericardium (Figure 2, $B$ and $C$ ). The SVC was reconstructed with bovine pericardium fashioned into a tube using a double row of staples (US Surgical Corporation, Norwalk, Conn) (Figure 2,D). The distal end of the graft was anastomosed in an end-to-end fashion to the SVC. The proximal end of the tube graft was anastomosed to a transverse opening in the right atrial pericardial patch. The pericardium was reconstructed with porcine submucosal tissue grafts (Cormatrix; Cormatrix Cardiovascular, Atlanta, Ga) to 